\title{
PSEUDOANGIOMATOUS STROMAL HYPERPLASIA OF BREAST: A CASE REPORT
}

\author{
Sachin Prasad Na*, Dana Houserkovaa ${ }^{a}$, Ivan Svach ${ }^{\mathrm{b}}$, Nora Zlamalova ${ }^{\mathrm{b}}$, \\ Ladislava Kucerova $^{\mathrm{c}}$, Karel Cwiertka ${ }^{\mathrm{d}}$
}

\author{
a Department of Radiology, Faculty of Medicine and Dentistry, Palacky University, 77515 Olomouc, Czech Republic \\ ${ }^{b}$ Department of Surgery, Faculty of Medicine and Dentistry, Palacky University \\ c Department of Pathology, Faculty of Medicine and Dentistry, Palacky University \\ d Department of Oncology, Faculty of Medicine and Dentistry, Palacky University \\ e-mail:drsachinprasadn@yahoo.co.in
}

Received: November 28, 2007; Accepted (with revisions): March 27, 2008

Keywords: Breast masses/Nodular tumour/Pseudoangiomatous stromal hyperplasia

Aim: To present a case of pseudoangiomatous stromal hyperplasia (PASH) and its findings under 1. mammography - MG, 2. ultrasonography - USG and 3. magnetic resonance imaging - MRI.

Materials and methods: A woman 39 years of age with a history of mass in her right breast of 3 months duration was subjected to a routine examination of the mass using MG \& USG. According to the modality findings a core cut biopsy was done following which the samples were send for histological analysis. Later, MRI was done as advocated by the surgeon to get a better picture of the extent of the lesion prior to surgery.

Results: Bilateral mammogram views revealed in the patient's right breast a huge well-bordered tumour of lobulated contour without halo sign. Sonography revealed a big well-demarcated tumour in the central part of the right breast which was cystic and lobulated in shape. Histological analysis of the sample confirmed pseudoangiomatous stromal hyperplasia (PASH). MRI under a breast array coil revealed a mass of $85 \times 75 \times 35 \mathrm{~mm}$ in the right breast. Finally, based on the clinical, radiological and histological report the mass was diagnosed as benign and despite the massive size of the mass, tumour excision alone was done and not mastectomy. The right breast after the huge tumour excision was almost normal in size compared to the left.

Conclusion: PASH should be included in the differential diagnosis of a circumscribed or partially circumscribed mass, especially in the pre-menopausal female population. These benign masses often grow over time and can recur locally. Radiological diagnosis of PASH is usually done by MG and USG followed by core cut biopsy for histological analysis. However great the mass is, excision only of the tumor mass is recommended and not mastectomy.

\section{INTRODUCTION}

Pseudoangiomatous stromal hyperplasia (PASH) of the breast is a rare benign proliferation of mesenchymal stromal cells with irregular slit-like formations resembling angiomatous structures ${ }^{1}$. In the majority of cases, this lesion is a focal microscopic finding in breast biopsies performed for benign or malignant diseases. It may present in a pure diffuse or nodular form. PASH is a localized fibroblastic and myofibroblastic overgrowth that occurs almost exclusively in premenopausal women as a painless, palpable intramammary mass ${ }^{1-3}$. It is a relatively common incidental finding in breast tissue removed for other reasons and rarely presents as a localized mass. The lesion is pale, fibrous, and has a homogeneous cut surface and is typically well circumscribed. Its ramifying slits lined by flattened myofibroblastic cells are apt to be mistaken for vascular spaces, leading to an erroneous diagnosis of angiosarcoma ${ }^{3}$. Pseudoangiomatous stromal hyperplasia (PASH) of the breast is a lesion characterized on histology by the presence of anastomosing slit like spaces embedded in a hyalinized fibrous stroma. However, the exact etiology and pathogenesis of this tumor-like lesion is still unknown, but a proliferative response of myofibroblasts to hormonal stimuli has been postulated ${ }^{2}$. Hormonal factors too are thought to play a developmental role.

\section{MATERIALS AND METHODS}

A woman 39 years of age, reported to our hospital with a history of mass in her right breast of 3 months duration which she noticed after the period of lactation for her third child. The patient had never taken any oral contraceptives. She was otherwise healthy without any other significant past or current medical problems. Physical examination of the patient suggested a mass in her right breast. The lymph nodes were not palpable. The skin overlying the mass, nipple and areola was normal and intact. Her left breast was normal. She was subjected to a routine examination of the mass using MG \& USG. Bilateral mammography was performed (Diamond, Instrumentarium Imaging, Tuusula, Finland) and included craniocaudal and mediolateral oblique view of the breasts. US using a linear-array transducer with a center frequency of 8-12 MHz was performed using Logiq $500 \mathrm{MD}$, General Electric, Solingen, Germany. The findings under these modalities were correlated. Later, according to the 


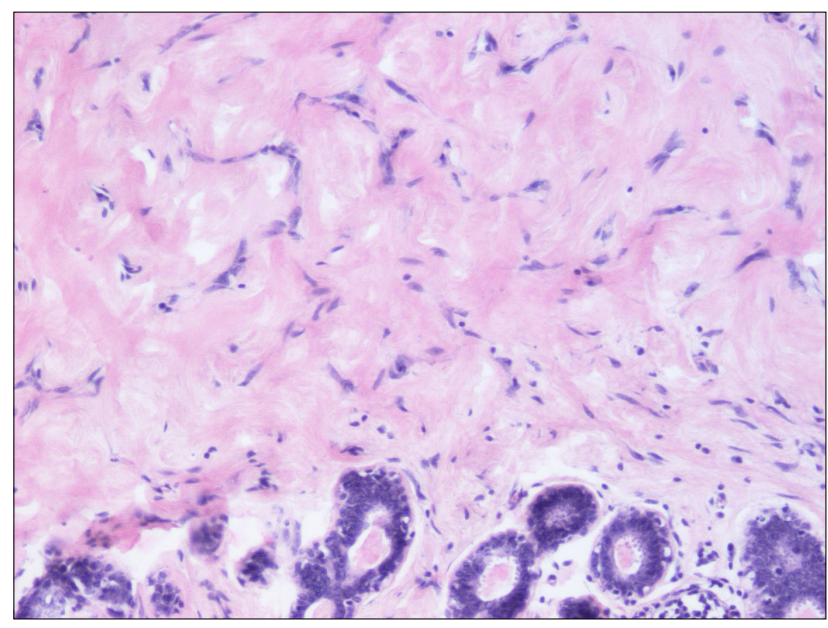

Fig. 1. Hematoxylin and eosin showing the stroma with intersection of pseudocapillaries and also the small ducts in the periphery.

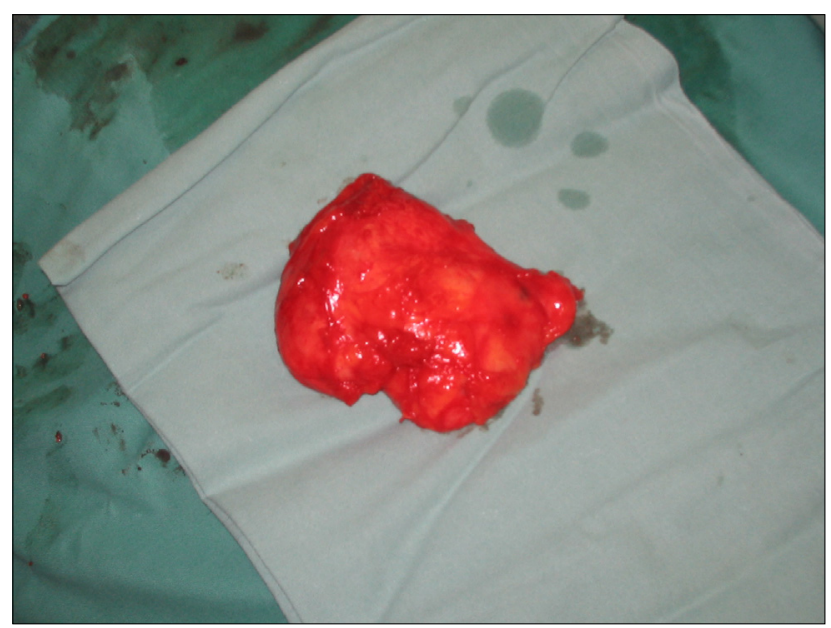

Fig. 3. The whole tumor after surgery. The tumour is encapsulated and is of lobulated shape.

modality findings a core cut biopsy was demanding and was performed under USG control after a consent was received from the patient following which the samples were then send for histological analysis. Histological diagnosis was determined by two pathologists with 10-20 years of experience in breast histology. MRI (magnetic resonance imaging) was also done as advocated by the surgeon to get a better picture of the extent of the lesion. MRI - native, T2 TSE, STIR and T1 fat saturation sequences in transverse and coronal plane were also performed. MRI was done using Siemens Symphony, Erlangen, Germany, Siemens. 1.5 Tesla under breast array coil. Finally, the clinical, radiological and histological reports were thoroughly analysed. A tumour excision was recommended and the tumor area was approached by an incision made at the periareolar region.

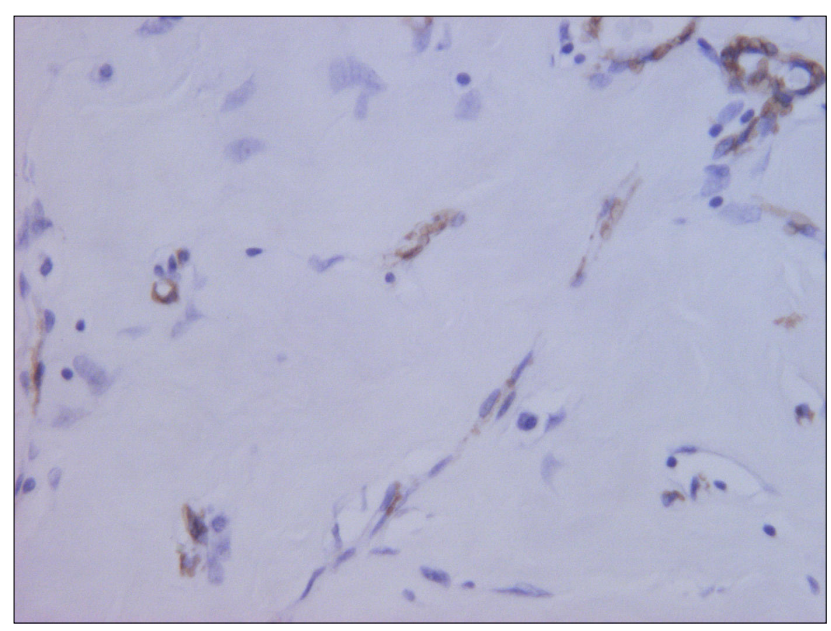

Fig. 2. Smooth muscle actin.



Fig. 4. Image of the excised tumour. The black spots inside the tumour correspond to small cystic areas.

\section{RESULTS}

Bilateral mammogram views revealed in the patient's right breast a huge well-bordered tumour of lobulated contour without halo sign but no evident calcifications. Sonography revealed a big well-demarcated tumour in the central part of the right breast which was cystic and approximately $75 \mathrm{~mm}$ in size and lobulated in shape. USG guided biopsy sample after histological analysis confirmed pseudoangiomatous stromal hyperplasia (PASH).

MRI under a breast array coil revealed a mass of $85 \times 75 \times 35 \mathrm{~mm}$ in the right breast. MRI - native, T2 TSE, STIR and T1 fat saturation sequences in transverse and coronal plane were performed. The tumour was seen to be located in the central retroareolar area of the right breast. The lesion was well demarcated and lobulated in shape. The signal intensity in native T2 was inhomogeneous and mainly hypointense. MRI-native, T1 3D spoiled gradient echo fat saturation pre-contrast image in transverse plane 
showed the tumour to be well-demarcated of benign appearance, and mainly of high intensity signal with hypointense septa. T1 3D spoiled gradient echo fat saturation $1^{\text {st }}$ post-contrast sequence image in transverse plane, revealed inhomogeneous enhancement of the tumour, with high signal intensity and small hypointense areas that correspond to small cystic lesions and hypointense septa. The time to intensity curve was of type-I (benign) with gradual uniform increase in signal intensity. With T1 3D spoiled gradient echo fat saturation 5 th post-contrast sequence in transverse plane, the whole tumour was seen clearly enhanced and only the septa remained hypointense. Finally, based on the clinical, radiological and histological report the mass was diagnosed as benign and despite the massive size of the mass, tumour excision alone was done and not mastectomy. MRI in these kind of patients is crucial in preventing total mastectomy. The right breast after the huge tumour excision was almost normal in size compared to the left. The excised tumour was well demarcated and had smooth external surface. The cut section of the tumour showed black spots which were the cystic area. After a couple of months the patient came for a routine check up and her breast was absolutely normal, with the disappearance of the periareolar scar.

\section{DISCUSSION}

Palpable breast masses are common and usually benign, but efficient evaluation and prompt diagnosis are necessary to rule out malignancy. A thorough clinical breast examination, imaging, and tissue sampling are needed for a definitive diagnosis. Breast masses have a variety of etiologies, benign and malignant. Most masses are benign, but breast cancer is the most common cancer and the second leading cause of cancer deaths in wom$\mathrm{en}^{1,5}$. An efficient and accurate evaluation can maximize cancer detection and minimize unnecessary testing and procedures. Pseudoangiomatous stromal hyperplasia (PASH) is a benign proliferative lesion of the mammary stroma containing complex anastomosing spaces that may be confused with angiosarcoma on histological analysis ${ }^{1}$. The tumoral form of PASH most commonly manifests as a single, circumscribed palpable mass in a pre-menopausal female ${ }^{1,2,6}$ as also seen with the patient in this study. The mass is usually large (5-6 cm in diameter), with reported diameters ranging from 1 to $12 \mathrm{~cm}$ (Ref., ${ }^{1,6}$ ). The reported age range of patients with PASH is 14-67 years, although most patients are in their forties or late thirties ${ }^{1,2,48}$ as also is the patient in this study. PASH has frequently been misdiagnosed on clinical examination as a fibroadenoma ${ }^{6,7}$. On mammography, masses due to PASH are usually noncalcified and appear well circumscribed or (as in this case) partially circumscribed. Indistinct or obscured borders have also been reported as has a single mass with a spiculated border ${ }^{2}$. The masses are seen at US as hypoechoic solid masses ${ }^{9}$. The echotexture may be slightly heterogeneous, and a small cystic component has been reported in one case ${ }^{9}$. Although mass lesions in PASH often grow over time and may recur after excision biopsy, they are neither associated with malignancy nor considered to be premalignant lesions ${ }^{6}$. At histologic analysis, this benign tumour of breast stroma has a "characteristic pattern of proliferating myofibroblasts that creates slit-like spaces" $1,7,8,10,11$. The myofibroblasts are believed to react aberrantly to hormones (particularly progesterone) that start as a focal accentuation of mammary physiologic changes during the menstrual cycle ${ }^{1,5,6}$. According to Powell et al. ${ }^{8}$, "Foci that eventually create discrete masses probably escape normal physiological control mechanisms, cease cycling with the remaining breast, and acquire the capacity for independent myofibroblastic proliferation." True masses are generally asymmetrical in relation to the other breast, distinct from the surrounding tissues and three-dimensional $^{12}$. A typical cancer may be firm, have indistinct borders and have attachments to the skin or deep fascia with dimpling or nipple retraction ${ }^{13}$. Benign lesions typically have discrete, well-defined margins and are mobile. Cysts cannot reliably be distinguished from solid breast masses by palpation. With respect to a palpable breast mass, other imaging techniques remain investigational. Several imaging techniques are commonly used in the evaluation of palpable breast masses. The use of multiple modalities in the diagnosis of palpable masses has been advocated as a measure to increase the true positive rate. Imaging modalities can be reassuring when the physical examination is not highly suspicious and follow-up is planned. However, a highly suspicious physical examination should prompt biopsy regardless of the imaging findings. As with all age-related guidelines, pertinent clinical factors such as family history should be used to determine appropriate patient care.

\section{CONCLUSION}

PASH should be included in the differential diagnosis of a circumscribed or partially circumscribed mass, especially in the pre-menopausal female population. These masses often grow over time and can recur locally. Radiological diagnosis of PASH is usually done by MG and USG imaging followed by core cut biopsy for histological analysis. Pathologic diagnosis of the lesion may be difficult unless the pathologist is aware of the presence of a mass lesion and appreciates the stromal changes characteristic of such a lesion. Regarding tumor removal, however great the benign mass is, excision only of the tumor mass is recommended and not mastectomy.

\section{REFERENCES}

1. Gow KW, Mayfield JK, Lloyd D, Shehata BM. Pseudoangiomatous stromal hyperplasia of the breast in two adolescent females. Am Surg. 2004; 70(7):605-8.

2. Castro CY, Whitman GJ, Sahin AA Pseudoangiomatous stromal hyperplasia of the breast. Am J Clin Oncol. 2002; 25(2):213-6.

3. Vicandi B, Jimenez-Heffernan JA, Lopez-Ferrer P, Ortega L, Viguer JM. Nodular pseudoangiomatous stromal hyperplasia of the breast. Cytologic features. Acta Cytol. 1998; 42(2):335-41. 
4. Okoshi K, Ogawa H, Suwa H, Saiga T, Kobayashi H. A case of nodular pseudoangiomatous stromal hyperplasia (PASH). Breast cancer. 2006; 13(4):349-53.

5. Iancu D, Nochomovitz LE. Pseudoangiomatous stromal hyperplasia: presentation as a mass in the female nipple. Breast J. 2001; 7(4):263-5.

6. Taira N, Ohsumi S, Aogi K, Maeba T, Kawamura S, Nishimura R, Takashima S. Nodular pseudoangiomatous stromal hyperplasia of mammary stroma in a case showing rapid tumor growth. Breast Cancer. 2005; 12(4):331-6

7. Cho N, Oh KK, Park KY, Noh TW. Sclerosing lobular hyperplasia: sonographic pathologic correlation. Eur Radiol. 2003; 13(7):1645 50 .

8. Powell CM, Cranor ML, Rosen PP. Pseudoangiomatous stromal hyperplasia (PASH). A mammary stromal tumor with myofibroblastic differentiation. Am J Surg Pathol. 1995; 19(3):270-7.
9. Cohen MA, Morris EA, Rosen PP, Dershaw DD, Liberman L, Abramson AF. Pseudoangiomatous stromal hyperplasia: mammographic, sonographic, and clinical patterns. Radiology. 1996; 198(1):117-20.

10. Günhan-Bilgen I, Memiș A, Ustun EE, Ozdemir N, Erhan Y.Sclerosing adenosis: mammographic and ultrasonographic findings with clinical and histopathological correlation. Eur J Radiol. 2002; 44(3):232-8.

11. Mercado CL, Naidrich SA, Hamele-Bena D, Fineberg SA, Buchbinder SS. Pseudoangiomatous stromal hyperplasia of the breast: sonographic features with histopathologic correlation Breast J. 2004; 10(5):427-32.

12. Polger MR, Denison CM, Lester S, Meyer JE.Pseudoangiomatous stromal hyperplasia: mammographic and sonographic appearances. AJR Am J Roentgenol. 1996; 166(2):349-52.

13. Poulton TB, de Paredes ES, Baldwin M. Sclerosing lobular hyperplasia of the breast: imaging features in 15 cases. AJR Am J Roentgenol. 1995; 165(2):291-4. 